\title{
Effects of Impeller Configuration on the Performance of a Double Suction Pump
}

\author{
Jeong-Min Jin', Yo-Hwan Kim ${ }^{1}$, Yeong Wan Je${ }^{1}$ and Youn-Jea Kim² \\ ${ }^{1}$ Graduate school of Mechanical Engineering, Sungkyunkwan University \\ Suwon 16419, Republic of Korea \\ wjdals3927@skku.edu; kyh8030@skku.edu \\ ${ }^{2}$ School of Mechanical Engineering, Sungkyunkwan University \\ Suwon 16419, Republic of Korea \\ yjkim@skku.edu
}

\section{Extended Abstract}

Depending on the number of inflow directions, the centrifugal pump can be classified as single suction pump in one direction or double suction pump in two directions. The characteristics of the two types of pumps are that the single suction pump has a high head while the double suction pump has a large flow rate. When the pump generates a pressure differential to rotate the impeller to transfer the working fluid, but the cavitation could be occurred consequently. Cavitation can cause vibration and noise, interfere with fluid flow and cause performance degradation and erosion of pump. Therefore, many studies have been continuing to improve pump performance and reduce cavitation [1 3]. The purpose of this study is to derive the optimal shape of inlet and outlet angles of the impeller and to improve the performance of the pump. Optimization of the impeller established 25 design points for the four design variables using the Central Composite Design (CCD), one of the Design of Experience (DOE) methods. Based on the results of the DOE, an optimal design point was obtained using the Response Surface Method (RSM). In addition, the Net Positive Suction Head (NPSH) used as a measure for safe operation of the pump without cavitation was obtained from the optimal and reference shapes and its results were used for evaluating the cavitation occurrence and structural stability. Numerical analysis was conducted using the commercial code ANSYS CFX ver. 18.1. The verification of the numerical results is compared with the existing experimental results for the reference model. Results show that the efficiency of the pump impeller was improved by $2.34 \%$ and the head by $0.72 \%$ compared with that of the reference model according to the change of the inlet and outlet angles of the pump impeller, and the influence of the impeller angle on the NPSH was insignificant.

\section{References}

[1] S. Hatano, D. Kang, S. Kagawa, M. Nohmi and K. Yokota, "Study of Cavitation Instabilities in Double-Suction Centrifugal Pump,” International Journal of Fluid Machinery and Systems, vol. 3, pp. 94-100, 2014.

[2] T. Shigemitsu, J. Fukutomi, R. Nasada and K. Kaji, "The Effect of Blade Outlet Angle on Performance and Internal Flow Condition of Mini Turbo-Pump,” Journal of Thermal Science, vol. 1, pp. 32-38, 2011.

[3] H. W. Oh, "Design Parameter to Improve the Suction Performance of Mixed-Flow Pump Impeller," in Proceedings of the Institution of Mechanical Engineers, Part A: Journal of Power and Energy, vol. 6, pp. 881-887, 2010. 